\title{
Research and correlation analysis on the dripper contamination of carteolol hydrochloride eye drops
}

\author{
Ying-Ming Wang ${ }^{1 \#}$, Ya-Lu Ren ${ }^{2 \#}$, Jie Xu ${ }^{2}$, Xiao-Feng Zhang ${ }^{1,3}$ \\ ${ }^{1}$ Department of Ophthalmology, the First Affiliated Hospital of Soochow University, Suzhou, China; ${ }^{2}$ Center of Clinical Laboratory, the First Affiliated \\ Hospital of Soochow University, Suzhou, China; ${ }^{3}$ Department of Ophthalmology, Dushu Lake Hospital Affiliated to Soochow University, Suzhou, China \\ Contributions: (I) Conception and design: YM Wang; (II) Administrative support: XF Zhang, J Xu; (III) Provision of study materials or patients: YM \\ Wang; (IV) Collection and assembly of data: YM Wang, YL Ren; (V) Data analysis and interpretation: YM Wang, YL Ren; (VI) Manuscript writing: \\ All authors; (VII) Final approval of manuscript: All authors. \\ \#These authors contributed equally to this work. \\ Correspondence to: Xiao-Feng Zhang. Department of Ophthalmology, the First Affiliated Hospital of Soochow University, Suzhou, China. \\ Email: zhangxiaofeng@suda.edu.cn.
}

\begin{abstract}
Background: Multi-dose eye drops are easily contaminated by microorganisms, and reportedly, the highest contamination rate can reach $96.46 \%$. The use of contaminated eye drops can cause serious eye infections.

Methods: Carteolol hydrochloride eye drops provided by glaucoma patients who visited the ophthalmic clinic of the First Affiliated Hospital of Soochow University from May 2018 to December 2019 were collected. Microbial culture was carried out on the eye drops, and the microbial species were identified by standard procedures. At the same time, the unsealing time, storage method, hand cleaning before dripping, and contact with the eyelid or the surrounding environment during infusion were recorded. Univariate and multivariate logistic regression analyses were used to analyze the risk factors associated with the contamination of carteolol hydrochloride eye drops.

Results: A total of 244 bottles of carteolol hydrochloride eye drops were collected, and the positive rate of flora culture was $6.6 \%$. A total of 18 strains of bacteria were isolated. The most common bacteria were Staphylococcus epidermidis and Corynebacterium. Univariate analysis showed that the risk factors associated with contamination were the unsealing time, the frequency of daily use, contact with the eyelid or the surrounding environment during the infusion process, and the use of more than 2 kinds of eye drops at the same time. Multivariate analysis showed that the unsealing time, the frequency of daily use, and contact with the eyelid or the surrounding environment were independent risk factors associated with contamination.
\end{abstract}

Conclusions: A long unsealing time, frequent use, and non-standard operation can increase the risk of eye drop contamination, which cannot be ignored.

Keywords: Glaucoma; eye drop; microbial contamination; risk factors

Submitted Apr 20, 2021. Accepted for publication Jun 11, 2021.

doi: 10.21037/apm-21-1237

View this article at: https://dx.doi.org/10.21037/apm-21-1237

\section{Introduction}

Multi-dose eye drops are easily contaminated by microorganisms in clinical application. Reportedly, the highest contamination rate of eye drops can reach
$96.46 \%$ (1). Contaminated eye drops can breed the growth of pathogenic or non-pathogenic microorganisms such as Staphylococcus epidermidis, Pseudomonas aeruginosa, Serratia marcescens, and yeasts (2). The use of such contaminated

$\wedge$ ORCID: 0000-0002-0628-0002. 
solutions can lead to serious eye infections, such as endophthalmitis, keratitis, and conjunctivitis, among others (3-5). At the same time, these microorganisms can also change the $\mathrm{pH}$ value, destroy the effective components, and affect the therapeutic effect $(6,7)$.

There are more than 70 million glaucoma patients worldwide, and the topical use of eye drops is still the basis of glaucoma treatment $(8,9)$. Glaucoma drugs are more likely to be contaminated compared with antibiotic eye drops with antibacterial capability (10). Previous studies have found that the risk factors associated with eye drop contamination include patients' age, unsealing time, use frequency, storage environment, and contact with the eyelid $(3,10-12)$, but the correlation of use time and frequency with the contamination rate is still controversial (13-15). The purpose of the current study was to investigate the contamination of multiple-dose carteolol hydrochloride eye drops and to identify the independent risk factors associated with contamination, so as to provide a theoretical basis for reducing the microbial contamination rate of eye drops and increasing the medication safety of glaucoma patients. We present the following article in accordance with the STROBE reporting checklist (available at https://dx.doi. org/10.21037/apm-21-1237).

\section{Methods}

\section{Sample collection}

Carteolol hydrochloride eye drops used by glaucoma patients who visited our hospital from May 2018 to December 2019 were collected. All patients reported that they used eye drops themselves. Gender, age, education level, visual acuity, intraocular pressure, glaucoma type, surgery history, and type of eye drops were recorded. The unsealing time of eye drops after opening, the storage method, hand cleaning before dripping, and contact with the eyelid or surrounding environment during infusion were recorded. All procedures performed in this study involving human participants were in accordance with the Declaration of Helsinki (as revised in 2013). The study was approved by Ethics Committee of the First Affiliated Hospital of Soochow University (NO.064) and informed consent was taken from all the patients.

\section{Specimen sampling and culture}

All obtained eye drop bottles were stored in sterile bags and refrigerated at $4{ }^{\circ} \mathrm{C}$, and samples were obtained within 24 hours. When sampling, the tip of the bottle was wiped with a sterile cotton swab soaked in sterile normal saline, then inoculated on a blood agar plate, chocolate plate, and Sabao weak plate, and incubated in a $35^{\circ} \mathrm{C}$ incubator for 24-72 hours (16). If the flat panel showed the growth of microorganisms, strain identification was carried out according to the standard laboratory procedure.

\section{Microbial identification}

The VITEK-MS automatic microbial mass spectrometer [Merier (China) Company] was used to identify bacteria or fungi: a 48-well target plate [Merier (China) Company] smear method was used, $\alpha$-cyano-4-hydroxycinnamic acid (CHCA) matrix solution [Merier (China) Company] was added, and ATCC8739 was used as the strain for quality control. The identification results were checked with the corresponding Myla software. The filamentous fungi which could not be identified by VITEK-MS were stained with phenol blue for morphological observation.

\section{Statistical analysis}

SPSS 21.0 software was used for data analysis. Count data were expressed by frequency and percentage, and the Chisquare test or Fisher's exact test were used for comparisons between groups. Measurement data were expressed by the mean \pm standard deviation or median and interquartile distance, and the $t$-test or non-parametric rank sum test were used for comparisons between groups. $\mathrm{P}<0.05$ was considered statistically significant.

\section{Results}

A total of 244 bottles of carteolol hydrochloride eye drops provided by 244 glaucoma patients were collected. Among the volunteers, 118 were male and 126 were female. There were 98 patients under 60 years old and 146 patients over 60 years old. There were 118 patients with primary angle closure glaucoma, 99 patients with primary open angle glaucoma, 4 patients with residual glaucoma, and 25 patients with secondary glaucoma (Table 1).

\section{Microbial culture}

Among the 244 eye drops, 16 eye drops were contaminated by 1 or more microorganisms, yielding a contamination rate 
Table 1 General information of the providers of carteolol hydrochloride eye drops

\begin{tabular}{lcc}
\hline General data & Subtypes & Value \\
\hline Gender & Male & 118 \\
\multirow{2}{*}{ Age } & Female & 126 \\
& $<60$ & 98 \\
Diagnosis & $\geq 60$ & 146 \\
& Primary angle closure glaucoma & 118 \\
& Primary open angle glaucoma & 99 \\
& Residual glaucoma & 4 \\
& Secondary glaucoma & 25 \\
\hline
\end{tabular}

Table 2 Microbial types and proportions

\begin{tabular}{llc}
\hline Microbial type & $\mathrm{n}$ & Proportion (\%) \\
\hline Staphylococcus epidermidis & 3 & 16.7 \\
Corynebacterium & 3 & 16.7 \\
Escherichia coli & 2 & 11.1 \\
Brevibacillus brevis $^{1}$ & 2 & 11.1 \\
Micrococcus $_{\text {Staphylococcus aureus }}$ & 1 & 5.5 \\
Klebsiella pneumoniae & 1 & 5.5 \\
Proteus mirabilis $^{1}$ & 1 & 5.5 \\
Bacillus badius $^{1}$ & 1 & 5.5 \\
Bacillus cereus $_{\text {Aspergillus sydowii }}^{2}$ & 1 & 5.5 \\
Paecilomyces variotii $^{2}$ & 1 & 5.5 \\
\hline
\end{tabular}

${ }^{1}$, mixed contamination of $B$. chestnut/B. brevis; ${ }^{2}$, mixed contamination of Aspergillus sydowii/Paecilomyces variotii.

of $6.6 \%$ (Table 2). Among the 16 contaminated eye drops, 14 $(87.5 \%)$ were contaminated by a single microorganism, and $2(12.5 \%)$ were contaminated by 2 kinds of microorganisms at the same time. A total of 18 strains of microorganisms were detected, including 16 strains of bacteria $(88.9 \%)$ and 2 strains of fungi (11.1\%). Staphylococcus epidermidis and Corynebacterium were the most common contaminating bacteria, accounting for $3 / 18(16.7 \%)$ of the total positive bacteria, while Escherichia coli and Brevibacillus brevis accounted for $2 / 18$ (11.1\%). Two cases of mixed contamination were Bacillus badius and Brevibacillus brevis, and Aspergillus sydowii and Paecilomyces variotii.

\section{Univariate analysis of the risk factors associated with dripper contamination of carteolol hydrochloride eye drops}

Univariate analysis showed that the unsealing time of eye drops, the frequency of daily use, contact with the eyelid or the surrounding environment in the process of infusion, and the use of more than 2 kinds of eye drops at the same time were the risk factors associated with dripper contamination of carteolol hydrochloride eye drops $(\mathrm{P}<0.05)$, as shown in Table 3.

\section{Multivariate analysis of the risk factors associated with dripper contamination of carteolol hydrochloride eye drops}

Multivariate logistic regression analysis showed that the unsealing time, the frequency of daily use, and contact with the eyelids or the surrounding environment of eye drops were independent risk factors associated with dripper contamination of carteolol hydrochloride eye drops $(\mathrm{P}<0.05)$, as shown in Table 4.

\section{Discussion}

The dripper is the component of eye drops which has the closest contact with the eyelid or surrounding environment. In addition, the spiral design inside the eye drop bottle cap and the wet dripper provide protection for the contaminated microorganisms, which may be the reason for the high contamination rate of the dripper (3). In this study, the contamination rate of eye drops was $16 / 244$ (6.6\%), which was lower than other reports on the contamination of glaucoma eye drops $(10,12)$. There were 2 cases of mixed contamination. One case was bacterial mixed contamination (Bacillus badius/Brevibacillus brevis), while the other case was fungal mixed contamination (Aspergillus sydowii/Paecilomyces variotii), and reports of contamination of 2 or more microorganisms have also been common $(10,13)$. In previous reports, symbiotic bacteria in the eyes and the surrounding environment became the dominant contamination microbial (17-19). In the current study, Staphylococcus epidermidis, bacillus, Micrococcus, Corynebacterium, and Staphylococcus aureus were all resident gram-positive bacteria in the eyelid and conjunctiva (3), while Escherichia coli and Proteus mirabilis were from soil or fecal contamination (1).

The types and sources of bacteria from the contaminated 
Table 3 Univariate analysis of the risk factors associated with dripper contamination of carteolol hydrochloride eye drops

\begin{tabular}{|c|c|c|c|c|c|c|}
\hline Factors & Subtypes & Total cases $(n)$ & $\begin{array}{c}\text { Contaminated } \\
\text { cases }(n)\end{array}$ & $\begin{array}{l}\text { Contamination } \\
\text { rate }(\%)\end{array}$ & $\chi^{2}$ & $\mathrm{P}$ \\
\hline \multirow[t]{2}{*}{ Gender } & Male & 118 & 10 & 8.5 & 1.371 & 0.242 \\
\hline & Female & 126 & 6 & 4.8 & & \\
\hline Age & $<60$ & 98 & 6 & 6.1 & 0.051 & 0.822 \\
\hline \multirow{2}{*}{ Education level } & Secondary school & 60 & 4 & 6.7 & & \\
\hline & University education & 72 & 4 & 5.6 & & \\
\hline Unsealing time (weeks) & $\leq 4$ & 151 & 5 & 3.3 & 6.813 & 0.009 \\
\hline \multirow[t]{2}{*}{ Contact history } & Yes & 78 & 10 & 12.8 & 7.340 & 0.007 \\
\hline & No & 166 & 6 & 3.7 & & \\
\hline \multirow[t]{2}{*}{ Drug type } & $\leq 2$ & 165 & 7 & 4.2 & 4.457 & 0.035 \\
\hline & $>2$ & 79 & 9 & 11.4 & & \\
\hline \multirow[t]{2}{*}{ Frequency of daily use } & Twice/day & 113 & 3 & 2.7 & 5.231 & 0.022 \\
\hline & 4 times/day & 131 & 13 & 9.9 & & \\
\hline Surgery history & Yes & 42 & 2 & 4.8 & 0.267 & 0.605 \\
\hline
\end{tabular}

Table 4 Multivariate logistic regression analysis of the risk factors associated with dripper contamination of carteolol hydrochloride eye drops

\begin{tabular}{|c|c|c|c|c|c|c|}
\hline Factor & $\beta$ & SE & Wald & $P$ & OR & $95 \% \mathrm{Cl}$ \\
\hline Frequency of daily use & 1.418 & 0.672 & 4.453 & 0.035 & 4.128 & $1.106-15.407$ \\
\hline Contact history & -1.157 & 0.562 & 4.239 & 0.040 & 0.314 & $0.104-0.946$ \\
\hline
\end{tabular}

drippers illustrated the fact that the main route of eye drop contamination was contact with the eyelid or the surrounding environment. During the use of eye drops, $78 / 244(32 \%)$ patients had a contact history with the eyelids, eyelashes, or conjunctiva, which was consistent with the results reported in other literature $(20,21)$. Previous studies showed that the poor infusion capability of glaucoma patients was related to age, limited education, and poor hands-on flexibility $(22,23)$. In the current study, the contamination rate was not associated with the age and education level of glaucoma patients. In addition, only $27.5 \%$ of glaucoma patients reported hand washing and other hand cleaning procedures, although the actual effect of hand cleaning remains to be verified. In the current report, $42 / 244(17.2 \%)$ patients had a history of unilateral or bilateral eye surgery, including glaucoma surgery, 
cataract surgery, and vitrectomy. However, unfortunately, the experience of such surgery did not improve the health awareness of patients, and there was no statistical difference in the contamination rate of drippers between the operation group and the non-operation group. Choy et al. (23) reported that the infusion success rate can be significantly improved after eye drop skills training in a group of elderly patients. Thus, some specially-designed eye drop bottles $(24,25)$ or special eye drop aid systems $(26)$ may play a vital role in addition to improving the infusion skills of eye drops and improving the safety awareness of hand hygiene.

Teuchner et al. (10) conducted microbial culture on eye drops used in patients' homes, the outpatient clinic, the ward, and the operating room, and found that the contamination rate of eye drops used in the operating room was the lowest, but there was no difference in the contamination rate of eye drops in the other 3 areas. In the current study, according to the different storage environments, carteolol hydrochloride eye drops were divided into room temperature and cold storage $\left(4^{\circ} \mathrm{C}\right)$. No difference was observed when they were stored in the 2 environments. These results were consistent with $\mathrm{Su}$ et al.'s findings (27).

The unsealing time of eye drops was one of the risk factors associated with contamination. The longer the unsealing time, the higher the contamination risk. The positive correlation between the contamination rate and the unsealing time has been confirmed in many studies $(3,12,28)$. Multi-dose eye drops have a large capacity and long time span, which increases the contamination risk in the process of frequent use. In the current study, the contamination rate of carteolol hydrochloride eye drops used for $>4$ weeks was 4 times the rate when used for $<4$ weeks, and the difference was statistically significant $(\mathrm{P}<0.05)$. However, this correlation has not been found in some studies (14), which may be due to the inconsistency of the time limit of eye drops selected in different studies. Single-dose eye drops do not contain preservatives, and patients are usually required to use them within 24 hours, which can reduce the risk of microbial contamination (27).

In the current study, we found that the use frequency was another risk factor associated with contamination. The contamination rate was only $3 / 113(2.7 \%)$ for 2 times/day, and when the use frequency doubled (4 times/day), the contamination rate increased to $13 / 131$ (9.9\%), and the difference was statistically significant $(\mathrm{P}<0.01)$. According to the traditional concept, the more frequently the eye drops were used, the higher the contamination risk, which has been confirmed in many studies $(3,29,30)$. However, there have been controversial reports on the correlation between use frequency and contamination. Tamrat et al. (13) pointed out that the contamination rate of eye drops with lower use frequency was higher ( $<4$ times/day), and believed that for the same dose of eye drops, it tended to be used for a longer time, due to used less frequently, so they may be more vulnerable to contamination.

There were some limitations in the current study. First, vision was not included in the risk factor analysis as patients with poor vision was $\geq 20 / 200$ in the current study, and the patients self-reported no difficulty in the infusion process. Second, the small sample size of this study might have introduced selection bias. Third, this study only focused on the contamination of carteolol hydrochloride eye drops, and does not represent all glaucoma drugs.

\section{Conclusions}

The contamination risk of carteolol hydrochloride eye drops can be increased by using eye drops for a long time after opening, opening the eye drops frequently every day, and contacting the eyelids or the surrounding environment during the use of eye drops. For patients with poor infusion skills, the success rate of eye drop infusion can be effectively improved by gradual education and guidance. Single-dose eye drops (which do not contain preservatives) are usually required to be used within 24 hours, which can improve the safety of drug use.

\section{Acknowledgments}

Funding: None.

\section{Footnote}

Reporting Checklist: The authors have completed the STROBE reporting checklist Available at https://dx.doi. org/10.21037/apm-21-1237

Data Sharing Statement: Available at https://dx.doi. org/10.21037/apm-21-1237

Conflicts of Interest: All authors have completed the ICMJE uniform disclosure form (available at https://dx.doi. org/10.21037/apm-21-1237). The authors have no conflicts 
of interest to declare.

Ethical Statement: The authors are accountable for all aspects of the work in ensuring that questions related to the accuracy or integrity of any part of the work are appropriately investigated and resolved. All procedures performed in this study involving human participants were in accordance with the Declaration of Helsinki (as revised in 2013). The study was approved by Ethics Committee of the First Affiliated Hospital of Soochow University (NO.064) and informed consent was taken from all the patients.

Open Access Statement: This is an Open Access article distributed in accordance with the Creative Commons Attribution-NonCommercial-NoDerivs 4.0 International License (CC BY-NC-ND 4.0), which permits the noncommercial replication and distribution of the article with the strict proviso that no changes or edits are made and the original work is properly cited (including links to both the formal publication through the relevant DOI and the license). See: https://creativecommons.org/licenses/by-nc-nd/4.0/.

\section{References}

1. Kyei S, Appiah E, Ayerakwa EA, et al. Microbial safety implications of in-use topical diagnostic ophthalmic medications in eye clinics in Ghana. J Optom 2019;12:263-271.

2. Kyei S, France D, Asiedu K. Microbial contamination of multiple-use bottles of fluorescein ophthalmic solution. Clin Exp Optom 2019;102:30-34.

3. Tsegaw A, Tsegaw A, Abula T, et al. Bacterial Contamination of Multi-dose Eye Drops at Ophthalmology Department, University of Gondar, Northwest Ethiopia. Middle East Afr J Ophthalmol 2017;24:81-6.

4. Mah-Sadorra JH, Najjar DM, Rapuano CJ, et al. Serratia corneal ulcers: a retrospective clinical study. Cornea 2005;24:793-800.

5. Penland RL, Wilhelmus KR. Stenotrophomonas maltophilia ocular infections. Arch Ophthalmol 1996;114:433-6.

6. Fazeli MR, Nejad HB, Mehrgan H, et al. Microbial contamination of preserved ophthalmic drops in outpatient departments: possibility of an extended period of use. DARU J Pharmaceut Sci 2004;12:151-5.

7. Perry HD, Donnenfeld ED. Issues in the use of preservative-free topicals. Manag Care 2003;12:39-41.
8. He S, Stankowska DL, Ellis DZ, et al. Targets of Neuroprotection in Glaucoma. J Ocul Pharmacol Ther 2018;34:85-106.

9. Bluwol E. Glaucoma treatment. Rev Prat 2016;66:508-13.

10. Teuchner B, Wagner J, Bechrakis NE, et al. Microbial contamination of glaucoma eyedrops used by patients compared with ocular medications used in the hospital. Medicine (Baltimore) 2015;94:e583.

11. Clark PJ, Ong B, Stanley CB. Contamination of diagnostic ophthalmic solutions in primary eye care settings. Mil Med 1997;162:501-6.

12. Geyer O, Bottone EJ, Podos SM, Schumer RA, Asbell PA. Microbial contamination of medications used to treat glaucoma. Br J Ophthalmol 1995;79:376-9.

13. Tamrat L, Gelaw Y, Beyene G, et al. Microbial Contamination and Antimicrobial Resistance in Use of Ophthalmic Solutions at the Department of Ophthalmology, Jimma University Specialized Hospital, Southwest Ethiopia. Can J Infect Dis Med Microbiol 2019;2019:5372530.

14. Feghhi M, Mahmoudabadi AZ, Mehdinejad M. Evaluation of fungal and bacterial contaminations of patient-used ocular drops. Med Mycol 2008;46:17-21.

15. Livingstone DJ, Hanlon GW, Dyke S. Evaluation of an extended period of use for preserved eye drops in hospital practice. Br J Ophthalmol 1998;82:473-5.

16. Aptel F, Masset H, Burillon C, et al. The influence of disease severity on quality of eye-drop administration in patients with glaucoma or ocular hypertension. Br J Ophthalmol 2009;93:700-1.

17. Porges Y, Rothkoff L, Glick J, et al. Sterility of glaucoma medications among chronic users in the community. J Ocul Pharmacol Ther 2004;20:123-8.

18. Lee SS, Chen YS, Tsai HC, et al. Predictors of septic metastatic infection and mortality among patients with Klebsiella pneumoniae liver abscess. Clin Infect Dis 2008;47:642-50.

19. Nentwich MM, Kollmann KH, Meshack J, et al. Microbial contamination of multi-use ophthalmic solutions in Kenya. Br J Ophthalmol 2007;91:1265-8.

20. Hosoda M, Yamabayashi S, Furuta M, et al. Do glaucoma patients use eye drops correctly? J Glaucoma 1995;4:202-6.

21. Stone JL, Robin AL, Novack GD, et al. An objective evaluation of eyedrop instillation in patients with glaucoma. Arch Ophthalmol 2009;127:732-6.

22. Tatham AJ, Sarodia U, Gatrad F, et al. Eye drop instillation technique in patients with glaucoma. Eye (Lond) 
2013;27:1293-8.

23. Choy BNK, Zhu MM, Pang JCS, et al. Factors Associated with Poor Eye Drop Administration Technique and the Role of Patient Education among Hong Kong Elderly Population. J Ophthalmol 2019;2019:5962065.

24. Davies IJ, Brown NH, Wen JC, et al. An upright eyedrop bottle: accuracy, usage of excess drops, and contamination compared to a conventional bottle. Clin Ophthalmol 2016;10:1411-7.

25. da Costa AX, Yu MCZ, de Freitas D, et al. Microbial Cross-contamination in Multidose Eyedrops: The Impact of Instillation Angle and Bottle Geometry. Transl Vis Sci Technol 2020;9:7.

26. Davies I, Williams AM, Muir KW. Aids for eye drop administration. Surv Ophthalmol 2017;62:332-45.

27. Su CY, Yang YC, Peng CF, et al. Risk of microbial

Cite this article as: Wang YM, Ren YL, Xu J, Zhang XF. Research and correlation analysis on the dripper contamination of carteolol hydrochloride eye drops. Ann Palliat Med 2021;10(6):6779-6785. doi: 10.21037/apm-21-1237 contamination of unit-dose eyedrops within twenty four hours after first opening. J Formos Med Assoc 2005;104:968-71.

28. Jokl DH, Wormser GP, Nichols NS, et al. Bacterial contamination of ophthalmic solutions used in an extended care facility. Br J Ophthalmol 2007;91:1308-10.

29. Yin VT, Weisbrod DJ, Eng KT, et al. Antibiotic resistance of ocular surface flora with repeated use of a topical antibiotic after intravitreal injection. JAMA Ophthalmol 2013;131:456-61.

30. Rahman MQ, Tejwani D, Wilson JA, et al. Microbial contamination of preservative free eye drops in multiple application containers. Br J Ophthalmol 2006;90:139-41.

(English Language Editor: C. Betlazar-Maseh) 